\title{
TERMES « ENDÉMIQUES » DANS LE SOUS-CODE LANGAGIER DE L'ÉGLISE CATHOLIQUE EN FRANÇAIS ET EN TCHÈQUE
}

\author{
Pavlina Žídková
}

\begin{abstract}
This paper focused on the terms own to the domaine of the Catholic Church in French and in Czech. These terms are called "endemic". After a review of the theoretic starting points, the paper describes the research in dictionnaries, specialized texts and the computer parallel corpus InterCorp, bringing a list of 131 French "endemic" terms and of their Czech equivalents. The Czech equivalents are not allways "endemic".

Keywords: term; endemic term; sub-code; specialized language; equivalent.

Résumé : Le présent article traite la question des termes propres au domaine de l'Église catholique en français et en tchèque. Ces termes sont appelés " endémiques ». Après la présentation des points de départ théoriques, l'article décrit la recherche des termes «endémiques " à partir les dictionnaires, les textes de spécialité et le corpus parallèle informatisé InterCorp, en présentant la liste de 131 termes « endémiques » français et de leur équivalents tchèques. Les équivalents tchèques ne sont pas toujours « endémiques » à leur tour.
\end{abstract}

Mots-clé : terme ; terme « endémique » ; sous-code ; langue de spécialité ; équivalent.

\section{Introduction}

Que nous considérions la variation linguistique liée à l'usage dans un domaine de spécialité comme une langue de spécialité ou un sous-code langagier, c'est entre autre leur propre nomenclature terminologique qui les fait distinguer d'autres langues de spécialité ou d'autres sous-codes. Robert Dubuc définit le terme comme « (...) l'appellation d'un objet propre à un domaine donné » (Dubuc 2002 : 33).

Comment comprendre ce que $c^{\prime}$ est un terme, notamment un terme des sciences humaines, ${ }^{1}$ appartenant uniquement à un domaine de spécialité ? À quels critères doit-il correspondre? Du point de vue comparatif, l'équivalent d'un terme propre à un domaine donné dans la langue cible, aura-t-il le même trait?

\footnotetext{
1 Cette question ne serait pas aussi pertinente pour les sciences naturelles et techniques où il ne faut pas mettre en question la corrélation du signifiant et du signifié du terme et du concept désigné. Donc où l'appartenance d'un terme à un domaine de spécialité devrait être claire même hors du contexte (comparer à Machová 1995: 143-144).
} 
L'objectif de notre travail était de trouver, en accord avec les critères adoptés, un nombre élevé des termes n'appartenant qu'à un seul domaine de spécialité et de leurs équivalents en tchèque. Nous avons travaillé avec le domaine thématique de l'Église catholique. ${ }^{2}$ Des moyens linguistiques employés dans d'autres confessions chrétiennes vont pour nous constituer d'autres langues de spécialité ou d'autres sous-codes. Des termes propres au domaine de l'Église catholique seront dans le présent article désignés comme termes « endémiques».

Après avoir décrit de plus près nos points de départ théoriques, la méthode et le corpus de notre recherche, nous présentons la liste des termes français classifiés comme « endémiques » dans le langage de l'Église catholique et de leurs équivalents tchèques.

\section{Points de départ théoriques}

\subsection{Langues de spécialité et sous-codes}

Jusqu'ici nous avons employé les notions de la langue de spécialité et du sous-code langagier comme synonymes. À cet endroit nous tenterons d'expliquer la conception du sous-code de plus près et nous allons justifier notre choix pour cette conception.

L'idée de ce que c'est une langue de spécialité n'est pas unie. Il y a des conceptions de la langue de spécialité en tant qu'une variété de la langue nationale (un inventaire spécifique des moyens linguistiques), des conceptions qui mettent le rapport d'égalité entre la langue de spécialité et les textes de spécialité et d'autres (Roelcke 1999 : 16-17 ; comparer à Cabré 1998 : 115-121).

Nous allons travailler avec la notion du sous-code (la conception de Jan Šabršula et de Jitka Svobodová, voir p. ex. Šabršula - Svobodová - Krejzová 1983 : 294-341) et ceci pour plusieurs raisons. En premier lieu, le sous-code est une entité de la langue (non de la parole), constituée par des unités (lexicales, grammaticales, phonétiques) non-linéaires. ${ }^{3}$ En même temps, ces unités peuvent être distribuées dans les (con-)textes variés. ${ }^{4}$ Ensuite, la langue de spécialité, en tant qu'une variété de la langue nationale, pourrait être perçue comme une partie du sous-code de spécialité (sous-code horizontal, Šabršula - Svobodová - Krejzová 1983 : 308-309). Un sous-code de spécialité contient aussi des moyens linguistiques employés dans les énoncés qui ne sont pas produits par les spécialistes (p. ex. théologiens, catéchistes) mais qui font l'objet de leur intérêt (p. ex. moyens linguistiques dans les textes bibliques, liturgiques, dans la communication pastorale, ${ }^{5}$ etc.). Le sous-code pourrait donc être perçu comme une notion plus large que celle de la langue de spécialité.

Pour définir si un lexème appartient à un sous-code de spécialité ou non, nous allons employer le critère du concept désigné. Des termes comme abbesse n. f. ("supérieure d'une abbaye », Feuillet $2010: 3$ ), pale n. f. (" un linge rigide et carré dont le prêtre recouvre le calice pendant la Messe », Feuillet $2010: 85$ ), baptiser qqn (« conférer le bap-

2 Nous nous occupons du langage de l'Église catholique dans le cadre de notre thèse de doctorat.

3 C'est à dire un sous-code de spécialité n'égale pas un texte de spécialité.

4 Par exemple les textes de l'Église catholique peuvent traiter des sujets de la vie humaine et sociale variés, voir p. ex. les chapitres de la constitution pastorale du Vatican II Gaudium et Spes (L'essor de la culture, La vie économico-sociale, La vie de la communauté politique, La sauvegarde de la paix et la construction de la communauté des nations, etc.). Donc ce texte du magistère de l'Église emploie des termes d'autres domaines de l'activité humaine, d'autres sous-codes horizontaux.

5 Des jargons font, eux aussi, partie des sous-codes horizontaux (Šabršula - Svobodová - Krejzová 1983 : 309-310). 
Termes « endémiques » dans le sous-code langagier de l'Église catholique en français et en tchèque

tême à. ", Dictionnaire Hachette langue française 2001 : 106), Immaculée Conception n. f. (" privilège selon lequel la Vierge Marie a été conçue sans le Péché originel », Feuillet 2010 : 60), qui désignent des personnes, objets, activités ou théories d'un domaine de spécialité vont appartenir au sous-code de l'Église catholique. Ces moyens linguistiques servent la communication aux sujets culturels et religieux. ${ }^{6}$

Par contre si nous considérons la salutation adieu (employée au sens profane) ou l'expression familière kyrielle (« longue suite de mots », de Kyrie Eleison, Dictionnaire Hachette langue française 2001 : 649), nous allons attribuer ces unités lexicales à la langue générale.

\subsection{Situation d'un terme par rapport à un domaine de spécialité}

Robert Dubuc considère que «Strictement [...] un terme n'appartient qu'à un seul domaine d'emploi. » (Dubuc 2002 : 35). Néanmoins il admet qu' au niveau formel un terme peut apparaître dans plusieurs domaines. Car il adopte des signifiés variés, il s'agit dans ce cas-là de plusieurs termes distincts (Dubuc 2002 : 35).

Thorsten Roelcke distingue le vocabulaire de la langue de spécialité (en tant qu'une variété de la langue nationale) et le vocabulaire des textes de spécialité. Quant au vocabulaire de la langue de spécialité, il le répartit dans quatre groupes : le vocabulaire qui appartient uniquement à une langue de spécialité concernée, le vocabulaire qui appartient à plusieurs langues de spécialité, le vocabulaire qui appartient à d'autres langues de spécialité mais qui pourtant apparaît dans les textes du domaine concerné. Le quatrième groupe est représenté par le vocabulaire non-spécialisé qui apparaît dans les textes du domaine donné (Roelcke 1999 : 52). Pour le vocabulaire des textes de spécialité, Roelcke propose un autre modèle. Il consiste à délimiter des termes appartenant uniquement à un seul domaine, à partir de la totalité du vocabulaire utilisé dans les textes de spécialité du domaine concerné (Roelcke 1999 : 53). Donc selon ce dernier modèle, un terme n'appartenant strictement qu'à un seul domaine de spécialité est employé uniquement dans les textes de spécialité du domaine concerné.

La conception du terme appartenant uniquement à un seul domaine de spécialité va correspondre à la conception de la langue de spécialité (et évidemment du terme) adoptée par le linguiste.

\subsection{Termes « endémiques » et « non endémiques » au catholicisme}

Nous appelons un terme propre à un domaine de spécialité terme " endémique ». En biologie, endémique " se dit d'une espèce (animale ou végétale) dont l'aire de répartition est peu étendue et bien limitée. » (Guerard 1989: 501).

En adoptant la conception du sous-code, le terme " endémique » dans un domaine sera propre au système terminologique de ce domaine et il va désigner des concepts propres à ce domaine. Ni son signifiant ni son signifié ne vont figurer dans le système terminologique d'un autre domaine de spécialité. Par contre, un terme " endémique » dans un domaine de spécialité peut être employé aussi dans d'autres textes que seulement dans les textes de spécialité du domaine concerné.

\footnotetext{
Arno Anzenbacher, spécialiste en éthique sociale, distingue cinq domaines des relations sociales : celui de la famille, celui des sciences et techniques, celui économique, celui de la politique et du droit et celui de la culture et de la religion (Anzenbacher 2004 : 8). Cette classification pourrait inspirer une répartition élémentaire des vocabulaires désignant des concepts de spécialités variées.
} 
Un terme « endémique » dans le sous-code du catholicisme (p. ex. encyclique n. f.) sera propre au domaine de la religion catholique. Il va naître dans les textes de spécialité du catholicisme (des traités théologiques) ou dans les textes source de la religion catholique (les textes liturgiques, les catéchismes, le code du droit canonique, ${ }^{7}$ les encycliques et $\mathrm{d}^{\prime}$ autres documents, notamment en provenance du magistère de l'Église). Un terme " endémique " dans le sous-code catholique peut apparaître dans d'autres genres de textes (p. ex. textes de d'autres spécialités, textes journalistiques aux sujets variés) sans perdre la marque d'être propre au domaine du catholicisme.

Pour la complexité notons que nous distinguons des termes « endémiques » par leur signifiant, des termes «endémiques " par leur signifié et des termes "non endémiques".

Le terme " endémique » par son signifiant désigne le même concept existant dans deux ou plusieurs sous-codes, néanmoins les signifiants sont variés, p. ex. sacrement des malades et onction des mains qui désignent plus ou moins le même concept, le premier dans le catholicisme, le second dans l'orthodoxie).

Le cas du terme « endémique » par son signifié sera celui où le signifiant sera partagé par deux ou plusieurs sous-codes, mais où un signifié sera propre à un seul sous-code, p. ex. acceptations différentes du conseil presbytéral $n$. $m$. dans le catholicisme et dans le protestantisme, comparer à Le Tourneau 2005 : 174). Ces termes existent formellement dans plusieurs sous-codes.

Un terme partagé avec son sifnifiant et son signifié par plusieurs sous-codes sera dans le présent article désigné comme "non endémique » au catholicisme (p. ex. Père n. m., Fils n. m. et Saint-Esprit n. m., les personnes de la Trinité n. f. pour l'ensemble du christianisme). Ce type de termes prendra sa source surtout dans la Bible.

Notons encore que nous allons négliger si un terme a subi une déterminologisation ${ }^{8}$ et il est utilisé, par extension, dans la langue générale. Par exemple bréviaire $\mathrm{n}$. $\mathrm{m}$. a deux signifiés :

(1) RELIG CATHOL livre contenant des offices que les clercs lisent chaque jour (2) FIG Livre dont on fait sa lecture habituelle (Dictionnaire Hachette langue française 2001 : 145).

Comme le deuxième signifié ne constitue pas avec le signifiant bréviaire un nouveau terme, il ne va pas nous empêcher à garder bréviaire parmi les termes " endémiques » au catholicisme.

\section{Méthodologie de la recherche des termes «endémiques»}

Pour délimiter des termes « endémiques » dans le sous-code de l’Église catholique en français et en tchèque nous avons parcouru plusieurs étapes. En premier lieu, il s'agissait du travail avec les dictionnaires spécialisés, avec les dictionnaires généraux et avec certains textes de spécialité. En deuxième lieu, nous avons utilisé le corpus parallèle informatisé InterCorp.

\subsection{Les dictionnaires et les textes de spécialité}

Le dictionnaire de départ était le glossaire français du lexique chrétien Vocabulaire du christianisme (Feuillet 2010). Cet ouvrage de cent vingt-sept pages contient des entrées

\footnotetext{
7 Le Catéchisme de l'Église catholique sera dans le présent article désigné par le sigle CEC (pour la version française) et KKC (pour la version tchèque). Version tchèque du Code de droit canonique sera raccourci par le sigle CIC.

8 Pour la déterminologisation voir p. ex. Šabršula 1985 : 12.
} 
Termes «endémiques » dans le sous-code langagier de l'Église catholique en français et en tchèque

désignant des concepts de la théologie, de la liturgie, des beaux-arts et d'autres. On y trouve de même des phrasèmes employés dans la vie de l'Église, des sigles ou des appellations de certains ordres religieux. Une acception différente du même terme dans les confessions chrétiennes variées y est signalée.

Tout d'abord nous avons choisi à peu près neuf cents termes de cet ouvrage, en omettant des phrasèmes (comme habemus papam), des lexèmes de caractère non terminologique (p. ex. défroqué) et des noms propres des ordres religieux (sauf quelques variantes des appellations de certains ordres, comme Frères mineurs ou Frères prêcheurs). Grâce au dictionnaire de la théologie dogmatique (Rahner - Vorgrimler 1970), au Catéchisme de l'Église catholique (CEC) et à un manuel du droit canonique (Le Tourneau 2002) nous avons joint à notre corpus une autre cinquantaine d'unités terminologiques. Le corpus de départ a donc été représenté par à peu près neuf cent cinquante termes. Ce corpus a été réparti, préalablement, en quatre groupes : termes « endémiques », termes « endémiques » par leur signifiant, termes « endémiques » par leur signifié et termes «non endémiques » au catholicisme. Dans un premier temps, les termes « endémiques » ont représenté à peu près un cinquième du corpus des termes examinés.

À cette étape, il y avait deux critères pour confirmer un terme comme « endémique ». Premièrement, il fallait vérifier que le terme n'existe pas formellement dans plusieurs sous-codes (qu'il ne désigne pas des concepts de plusieurs sous-codes). C'est pourquoi nous avons travaillé avec les dictionnaires de la langue française (Grand Robert 2005 ; Dictionnaire Hachette langue française 2001 ; Guerard 1989). Pour certains termes, les dictionnaires disposaient de la marque religion catholique (par ex. rogations n. f. pl., Guerard 1989 : 1322).

Si ce n'était pas le cas, il fallait vérifier si ce terme n'était pas propre à un autre domaine encore. Par d'autres mots, s'il ne désignait pas un concept propre à un autre domaine qu'à celui de la religion catholique.

Dans ce but nous nous sommes servie de trois sources. Premièrement, du dictionnaire des termes du chritianisme (Le Tourneau 2005). Il s'agit d'un ouvrage volumineux, de 742 pages, donnant des informations sur l'étymologie et l'histoire des termes ainsi que sur leurs signifiés, selon les différentes confessions chrétiennes.

Deuxièmement, étant donnée la proximité de la liturgie catholique avec l'Église orthodoxe ou vu des parallèles de certaines institutions catholiques avec l'anglicanisme, c'étaient aussi quelques sources complémentaires, donnant des informations sur d'autres confessions qui nous ont parfois aidé à reclasser un terme " endémique » comme " non endémique » au catholicisme (p.ex. les sites web Lexique orthodoxe 2004 ou Wikipédia l'encyclopédie libre. Anglicanisme 2013)

Troisièmement, nous avons cherché des termes préalablement classifiés comme « endémiques » dans le corpus parallèle informatisé InterCorp.

\subsection{Le corpus parallèle informatisé InterCorp}

Afin de pouvoir travailler avec des termes, préalablement classifiés en français comme " endémiques » de manière efficace dans les textes, et afin de vérifier en même temps les équivalents tchèques, nous avons travaillé avec le corpus parallèle informatisé InterCorp, en version actuelle 6 (http:/ / www.korpus.cz/intercorp, en ligne le 29 mai 2013).

Avec l'intention de travailler avec le plus de sources possibles nous avons choisi tous les textes non artistiques disponibles pour le travail parallèle avec la langue française et tchèque. Il s'agissait de quatre paquets de textes. Les paquets Projet Syndicate et Presseurope 
représentent un recueil des articles journalistiques des sites web, le paquet Acquis contient les textes judiciaires de l'Union européenne (Acquis communautaire). De plus, le paquet des textes Europarl propose des enregistrements des discours du Parlement européen de 2007-2011. Donc, il ne s'agissait pas des textes propres au domaine de l'Église catholique. Quant aux textes littéraires, nous avons choisi l'œuvre du domaine de l'histoire ${ }^{9}$ de Georges Duby, Dames du XII siècle. Ce texte contient vraisemblablement de nombreuses informations culturelles sur la vie religieuse au Moyen Âge. Lors du choix des documents du corpus nous avons négligé s'il s'agissait d'un texte source français ou d'un texte traduit. Certes, sauf le paquet de textes Acquis, il ne s'agit pas de textes de spécialité au sens propre du mot. Néanmoins supposons que les termes y sont employés correctement.

En utilisant le moteur d'analyse PARK nous avons eu l'occasion de travailler avec un corpus de 50157264 mots $^{10}$ en français et avec un corpus parallèle de 37614570 mots en tchèque. Pour les termes examinés il s'agissait en grande partie (sauf quelques peu d'exceptions) des substantifs, que nous avons cherchés et au singulier et au pluriel. Pour chercher un terme à plusieurs mots, nous l'avons fait chercher en tant que slovní spojení " syntagme ». Les termes ont été cherchés à partir des textes français. Leur sens propre ou figuré a été examiné.

\subsection{Les équivalents tchèques}

Les équivalents tchèques des termes français classifiés comme "endémiques » ont été trouvés à l'aide des dictionnaires et des textes de spécialité. En travaillant avec les définitions des termes français, nous avons cherché dans les dictionnaires et les textes de spécialité tchèques les signifiants équivalents des termes français. Nous avons utilisé le dictionnaire encyclopédique de la liturgie (Berger 2008), le manuel des expressions religieuses sous forme de dictionnaire (Šimandl 2004), le dictionnaire de la théologie catholique (Fischer-Wollpert 1995) ou les textes du domaine (KKC, CIC, Franzen 1995).

Dans le pas suivant nous avons tenté d'équiper les équivalents tchèques par la marque " endémique » ou par une autre. Nous avons procédé de manière analogue comme dans le cas des termes français. Tout d'abord, nous nous sommes servie du dictionnaire des mots d'origine étrangère (Kraus 2005) et du dictionnaire de la langue tchèque (Havránek 1989) pour confirmer que le terme n'appartient pas formellement à d'autres domaines de spécialité. Ensuite c'étaient les ouvrages contenant la terminologie de d'autres confessions chrétiennes, comme le manuel du christianisme (Filipi 1998) ou le dictionnaire encyclopédique des églises protestantes (Filipi 2008) qui nous ont été bien utiles pour la classification des termes tchèques comme " endémiques » au catholicisme. Nous avons de même examiné des équivalents tchèques des termes " endémiques » français dans la partie tchèque du corpus parallèle InterCorp.

\section{Les termes «endémiques » dans le système des termes et dans 1'InterCorp}

\subsection{Les termes « endémiques » français}

Grâce aux dictionnaires et aux textes de spécialité du domaine choisi, nous avons répertorié une liste de 134 unités terminologiques avec la marque « endémique » au ca-

\footnotetext{
9 Il ne s'agit pas de l'œuvre scientifique, mais plutôt scientifique - vulgarisée.

${ }_{10}$ Le moteur Park a donné le résultat de la largeur du corpus choisi 50157264 slov (« mots ») pour la partie française du corpus, avec la remarque qu'il ne s'agissait pas du nombre précis.
} 
tholicisme. Nous en avons repéré 19 dans le corpus informatisé InterCorp, employées au moins une fois au sens propre (p. ex. béatifcation, ordres mendiants, surplis). Nous avons négligé l'emploi des termes au rôle des méthaphores et métonymies (déjà lexicalisées ou non), dont le langage religieux paraît être une source propice. Le nombre de termes propres au catholicisme trouvés dans l'InterCorp n'est pas élevé. Pourtant les termes recherchés ont été distribués au sens propre dans tous les types de textes choisis (Acquis, Europarl, Presseurop, Syndicate, Duby). Ceci démontre que les termes "endémiques" dans le sous-code catholique peuvent être employés correctement dans les textes de provenances et de genres variés.

Grâce à la recherche dans les textes de l'InterCorp nous avons éliminé de notre corpus de 134 termes " endémiques » trois unités. La raison était la désignation d'une entité propre à une religion différente que celle catholique (archiprêtre n. m., Sa sainteté n. f.) ou à un autre domaine thématique (vêture n. f.). Archiprêtre a désigné un ecclésiastique orthodoxe et le titre Sa sainteté a été attribué au Dalaï-lama. Le terme vêture signifie dans le langage catholique la cérémonie de prise d'habit religieux. Dans les textes d'Acquis il a désigné (employé au pluriel) un élément technique lors de la construction des bâtiments (paquet des textes Acquis, InterCorp 2013).

Le corpus résultant des termes français « endémiques » au catholicisme contient donc, selon nos recherches actuelles, 131 unités. Évidemment, vue la richesse terminologique du domaine choisi, ce corpus des termes « endémiques » au catholicisme n'est pas exhaustif.

Lors de notre recherche nous avons rencontré un certain problème, représenté par des termes concernant la religion et un autre domaine, p. ex. le catholicisme et l'histoire, comme pape n. m. ("Chef suprême de l'Église catholique romaine et évêque de Rome ", Dictionnaire Hachette langue française 2001 : 832). Est-il possible de qualifier un tel terme comme " endémique " au catholicisme ? Comme ce lexème désigne une entité ontologiquement propre à la religion catholique, notre réponse serait oui. Quant au domaine de l'histoire, pape pourrait être considéré comme un emprunt « interdisciplinaire ». ${ }^{11}$ Or, ce terme désigne aussi le «chef suprême de l'Église copte » (Guerard 1989 : 1104). Il en résulte que cette unité terminologique sera classifiée comme « endémique » dans le souscode catholique uniquement par un de ses signifiés.

Évidemment, des termes à plusieurs mots ont plus de chances d'être associés au groupe « endémique » dans le sous-code catholique (p. ex. instituts de vie consacrée $\mathrm{n} . \mathrm{m}$. pl., « ensemble de sociétés religieuses », Le Tourneau 2005 : 332).

Les signifiants des termes «endémiques » au catholicisme ne sont pas propres à d'autres sous-codes langagiers. Par contre, il est possible de repérer une polysémie au sein du seul sous-code en question (p. ex. Angélus n. m. en tant que prière à la Vierge et en tant que le son de cloche invitant à cette prière, Guerard 1989 : 57). Des termes au sein du groupe «endémique » peuvent de même être synonymes (p. ex. Saint-Siège n. m. et Siège apostolique n. m., Feuillet 2010 : 133).

\footnotetext{
${ }_{11}$ Pourtant dans le domaine de l'histoire pape serait employé et perçu plutôt avec l'accent sur son rôle du « chef des États du pape » que sur ses attributs du « successeur de saint Pierre, du vicaire de Jésus Christ », traits qui lui sont attribués par la théologie catholique (comparer à Grand Robert 2005).
} 


\subsection{Les équivalents tchèques dans le système de la langue et dans les textes de l'InterCorp}

Tout d'abord, il faut noter que parmi les 131 termes français « éndémiques » au catholicisme il y en a 10 dont nous n'avons pas trouvé l'équivalent direct dans le système des termes catholiques en tchèque. Il s'agit surtout des termes désignant des entités historiquement différentes dans les églises françaises et tchèques (p. ex. prêtres réfracteurs) et des appellations populaires de certaines cérémonies liturgiques (p. ex. communion privée).

Parmi les équivalents tchèques il y en avait 5 que nous avons classifiés comme «endémiques » par le signifié et 2 come " non endémiques ». Les premiers équivalents tchèques sont propres aussi à d'autres domaines de spécialité qu'à celui du catholicisme (p. ex. bekyně, n. f., terme propre par le signifiant à la religion catholique et à la biologie, comparer à Havránek 1989). Les termes « non endémiques » sont partagés par plusieurs confessions chrétiennes. Mentionnons parmi les termes " non endémiques» l'exemple farář n. m. L'équivalent du terme français curé $\mathrm{n}$. m. ("prêtre catholique à la tête d'une paroisse ", Grand Robert 2005) peut en tchèque désigner aussi le chef d'une paroisse évagélique. C'est-à-dire que le terme tchèque farár va désigner un concept concret de manière univoque ou bien à l'aide du contexte, ou bien accompagné d'un attribut (p. ex. farár ř rimsko-katolické farnosti « chef d'une paroisse catholique » ou bien farár Církve československé husitské " pasteur de l’Église tchécoslovaque hussite »).

Passons à la recherche du corpus InterCorp. En négligeant archiprêtre, Sa sainteté et vêture (4.1), parmi les 16 termes « endémiques » français restant, figurant dans les textes du corpus informatisé, nous avons repéré au moins une fois 14 termes équivalents tchèques - dans l'accord avec la recherche des équivalents à partir les dictionnaires spécialisés et les textes de spécialité.

Par exemple dans l'extrait de l'œuvre de Gerorges Duby Dames du XII sciècle I. nous trouvons le paire surplis n. m. - rocheta $\mathrm{n}$. f. :

(...) imposante assemblée d'hommes, de clercs en surplis, de chevaliers en arme/(...) působivé mužské shromáždění duchovních v rochetách a rytírư ve zbrani (InterCorp 2013).

\section{Termes français « endémiques " au catholicisme et leurs équivalents tchèques}

Selon l'état actuel de nos recherches, nous présentons ci-dessous la liste de 131 termes français « endémiques » au catholicisme et de leurs équivalents tchèques. Les abréviations entre parenthèses marquent l'appartenance du terme à un certain sous-domaine de spécialité. Pour les définitions, nous renvoyons aux dictionnaires spécialisés (Feuillet 2010 ; Le Tourneau 2005). Les équivalents tchèques non classifiés comme " endémiques » au catholicisme sont accompagnés par les abréviations epsé et ne (voir la liste des abréviations). L'espèce de mot et le genre grammatical est rapporté à l'élément de base de l'unité terminologique. L'équivalent tchèque non trouvé est signalé par le symbole $X$.

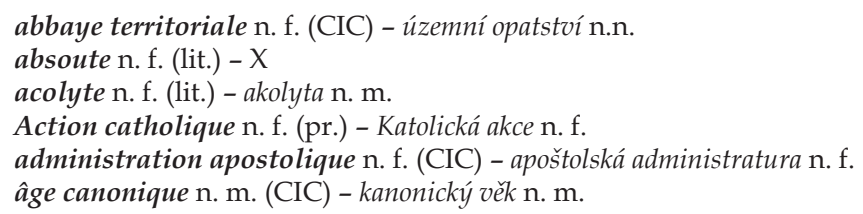


aggiornamento n. m. (mag.) - aggiornamento n. n.

Angélus n. m. (lit) - Anděl Páně n. m.

anneau pastoral $\mathrm{n}$. m. (lit.) - biskupský prsten $\mathrm{n}$. $\mathrm{m}$.

année sainte $\mathrm{n}$. $\mathrm{f}$. (pr.) - svatý rok $\mathrm{n} . \mathrm{m}$.

Assomption n. f. (lit.) - Nanebevzetí Panny Marie n. m.

basilique majeure (lit.) n. f. - basilica maior $\mathrm{n}$. f.

basilique mineur $\mathrm{n}$. f. (lit.). - basilica minor $\mathrm{n}$. f.

béatification $\mathrm{n}$. f. (mag.) - beatifikace $\mathrm{n}$. $\mathrm{f}$.

béguine $\mathrm{n} . \mathrm{f}$. (pr.) - bekyně n. f. (epsé)

bénédicité $\mathrm{n}$. $\mathrm{f}$. (lit.) - X

bréviaire $\mathrm{n} . \mathrm{m}$. (lit.) - breviář $\mathrm{n} . \mathrm{m}$.

canon de la messe n. m. (lit.) - mešní kánon $\mathrm{n}$. m.

canoniste $\mathrm{n}$. m. (CIC) - kanonista $\mathrm{n}$. $\mathrm{m}$.

casuistique n. f. (th.) - kazuistika n. f.

célébrant $\mathrm{n} . \mathrm{m}$. (lit.) - celebrant $\mathrm{n} . \mathrm{m}$.

cérémonie pénitentielle communautaire $\mathrm{n}$. f. (lit.) - společné slavení svátosti smírení

Chandeleur n. f. (lit) - Hromnice n. f. pl.

chant grégorien $\mathrm{n}$. m. (lit.) - gregoriánský chorál n. m.

chapelain n. m. (pr.) - kaplan n. m. (epsé)

ciboire n. m. (lit.) - ciborium n. n.

cloître n. m (pr.) - 1. klauzura n. f. (epsé), 2. klášter n.m. (ne), 3. kř́žzová chodba n. f.

collège des consultaires n. m. (pr.) - kolegium konzultorü n. n.

communion privée $\mathrm{n}$. $\mathrm{f}$. (lit.) - $\mathrm{X}$

communion solennelle $\mathrm{n}$. $\mathrm{f}$. (lit.) - $\mathrm{X}$

concil plénier $\mathrm{n}$. m. (CIC) - plenární sněm $\mathrm{n} . \mathrm{m}$.

conclave $\mathrm{n}$. $\mathrm{m}$. (pr.) - konkláve $\mathrm{n} . \mathrm{n}$.

conférences des évêques $\mathrm{n}$. f. pl. (CIC) - biskupské konference $\mathrm{n}$. $\mathrm{f}$. $\mathrm{pl}$.

conseil de fabrique $\mathrm{n}$. m. (pr.) - X

congrégation romaine $\mathrm{n}$. f.(mag., pr.) - ř́mská kongregace $\mathrm{n}$. f.

conseil épiscopal n. m. (pr.) - biskupská rada n. f.

conventuel $\mathrm{n} . \mathrm{m}$. (pr.) - františkán konventuál n. m.

convers, rse n. m., n. f. (pr.) - konvrš, konvrška n. m., n. f.

Corpus Domini n. m. (lit.) - Boží Tělo n. n.

curé n. m. (pr.) - farář n. m. (ne)

curie diocésaine $\mathrm{n}$. $\mathrm{f}$. (CIC) - diecézní kurie n. f.

curie romaine $\mathrm{n}$. $\mathrm{f}$. (CIC) - papežská kurie $\mathrm{n}$. f.

décrétale $\mathrm{n}$. f. (mag.) - dekretál n. m. (dekretále n. n.)

dimanche Latare n. m. (lit.) - neděle Laetare n. f.

droit canonique $\mathrm{n}$. m. (CIC) - kanonické právo n. $\mathrm{n}$.

docteur de l'Église n. m. (th.) - učitel církve n. m.

encyclique m. f. (mag.) - encyklika n. f.

épikeia n. f. (th.) - epikie n. f.

ex cathedra loc. adv. (mag.) - ex cathedra loc. adv.

excommunication $\mathrm{n}$. $\mathrm{f}$. (mag., CIC) - exkomunikace $\mathrm{n}$. $\mathrm{f}$.

fabricien n. m. (pr.) - X

Fête-Dieu n. f. (lit.) - Boží Tělo n. n.

frère lai $\mathrm{n} . \mathrm{m}$. (pr.) - bratr laik $\mathrm{n}$. $\mathrm{m}$.

Frères mineurs n. m. pl. (pr.) - Menší bratři n. m. pl.

Frères prêcheurs n. pl. m. (pr.) - Bratři kazatelé n. m. pl.

grand-messe $\mathrm{n}$. f. (lit.) - mše slavná $\mathrm{n}$. f.

Immaculée n. f. (th.) - Panna Maria počatá bez poskvrny hř́chu dědičného n. f.

Immaculée Conception n. f. (th.) - Neposkvrněné početí n. n.

immaculistes n. m. pl. (th.) - imakulisté n. m. pl.

infaillibilité pontificale n. $\mathrm{f}$. (th.) - papežská neomylnost $\mathrm{n}$. $\mathrm{f}$.

instituts de vie consacrée $\mathrm{n}$. m. pl. (CIC) - instituty zasvěceného života n. m. pl. 
introüt $\mathrm{n}$. $\mathrm{m}$. (lit.) - introit $\mathrm{n}$. $\mathrm{m}$.

litanies de la Vierge n. f. pl. (lit.) - litanie k Panně Marii n. f. pl.

liturgie de la Parole n.f. (lit.) - liturgie Slova n. f.

liturgie eucharisitque n. f. (lit.) - eucharistická liturgie n. f.

maculistes n. m. pl. (th.) - makulisté n. m. pl.

Mercredi des cendres n. m. (lit.) - Popeleční středa n. f.

messe basse n. f. (lit.) - mše tichá n. f.

missel n. m. (lit.) - misál n. m.

Mi-Carême n. f. (lit.) - X

mission canonique $\mathrm{n}$. $\mathrm{f}$. (CIC) - kanonická mise $\mathrm{n}$. $\mathrm{f}$.

monstrance n. f. (lit.) - monstrance n.f., ostenzorium n. n.

mosette n. f. (lit.) - mozzetta n. f.

neuvaine $\mathrm{n}$. f. (lit.) - novéna $\mathrm{n}$. $\mathrm{f}$.

nonce apostolique $\mathrm{n} . \mathrm{m}$. (CIC) - apoštolský nuncius $\mathrm{n} . \mathrm{m}$.

nouvelle évangélisation $\mathrm{n}$. $\mathrm{f}$. (mag.) - nová evangelizace $\mathrm{n}$. $\mathrm{f}$.

offertoire $\mathrm{n}$. m. (lit.) - ofertotium $\mathrm{n}$. $\mathrm{n}$.

officiant $\mathrm{n}$. m. (lit.) - celebrant $\mathrm{n}$. $\mathrm{m}$.

ordinaire de la messe n. m. (lit.) - mešní ordinarium n. n.

ordres majeurs n. m. pl. (th., lit.) - vyšší kněžská svěcení n. n. pl.

ordres mendiants n. pl. m. (pr.) - žebravé řády n. m. pl.

ordres mineurs n. m. pl. (th., lit.) - nižší kněžská svěcení n. n. pl.

ostensoir n. m. (lit.) - monstrance n. f., ostenzorium n. n.

pontifical n. m. (lit) - pontifikál n. m.

pourpe cardinalice n. f. (lit.) - kardinálský purpur n. m.

préfecture apostolique $\mathrm{n}$. $\mathrm{f}$. (CIC) - apoštolská prefektura $\mathrm{n} . \mathrm{f}$.

première communion $\mathrm{n}$. $\mathrm{f}$. (lit.) - proní svaté prijímání $\mathrm{n}$. $\mathrm{n}$.

prélat $\mathrm{n} . \mathrm{m} .(\mathrm{CIC})-$ prelát $\mathrm{n} . \mathrm{m}$.

prélature personnelle $\mathrm{n}$. $\mathrm{f}$. (CIC) - osobni prelatura $\mathrm{n}$. $\mathrm{f}$.

prélature terittoriale $\mathrm{n} . \mathrm{f}$. (CIC) - územní prelatura $\mathrm{n}$. $\mathrm{f}$.

Présentation de Jésus au Temple n. f. (lit.) - Uvedení Páně do Chrámu n. n.

prêtres réfracteurs $\mathrm{n}$. $\mathrm{m}$. pl. (pr.) $-\mathrm{X}$

prêtres-ouvriers n. m. pl. (pr.) - dělničtí kněží n. m. pl.

prie-Dieu n. m. (lit.) - klekátko n. n.

prieuré n. f. (pr.) - prevoorstoí n. $\mathrm{n}$.

prise d'habit/de voile n. f. (lit.) - obláčka n. f.

profès, sse n. m., n. f. (pr.) - profes, ka n. m., n. f.

profession religieuse $\mathrm{n}$. f. (lit.) - profese $\mathrm{n}$. f. (epsé)

purgatoire n. m. (th.) - očistec n. m.

Purification de la Vierge n. f. (lit.) - Očištování Panny Marie n. n.

quadragésime $\mathrm{n}$. f. (lit.) - quadragesima $\mathrm{n}$. $\mathrm{f}$.

quatre-temps n. m. pl. (lit.) - kvatembr n. m.

réforme grégorienne $\mathrm{n}$. f. (pr.) - gregoriánská reforma $\mathrm{n}$. f.

Règle de saint Benoît $\mathrm{n}$. f. (pr.) - Řehole svatého Benedikta n. f.

rogations $\mathrm{n}$. f. pl. (lit.) $-\mathrm{X}$

rosaire $\mathrm{n} . \mathrm{m}$.(lit). - růženec $\mathrm{n} . \mathrm{m}$.

Sacré collège n .m. (mag.) - posvátné kolegium kardinálů n. n.

sacristie n. f. (lit.) - sakristie n. f.

Saint-Office n. m. (mag.) - Svaté oficium n. m.

Saint-Sacrement n. m. (lit.) - Nejsvětější svátost (oltářní) n. f.

Saint-Siège n. m. (mag.) - Svatý stolec n. m.

Salve Regina n. m. (lit.) - Zdrávas Královno n. m.

sanctoral $\mathrm{n}$. m. (lit.) - sanktorál $\mathrm{n}$. $\mathrm{m}$.

septuagésime $\mathrm{n}$. f. (lit.) - septuagesima $\mathrm{n}$. f.

Siège apostolique n. m. (mag.) - Apoštolský stolec n. m.

sociétés de vie apostolique $\mathrm{n}$. f. pl. (CIC) - společnosti apoštolského života n. f. pl. 
somme théologique $\mathrm{n}$. $\mathrm{f}$. (th.) - teologická suma $\mathrm{n} . \mathrm{f}$

surplis $\mathrm{n}$. m. (lit.) - rocheta $\mathrm{n}$. $\mathrm{f}$.

synode diocésain $\mathrm{n} . \mathrm{m}$. (CIC) - diecézní sněm $\mathrm{n}$. $\mathrm{m}$.

théologie de la libération $\mathrm{n}$. f. (th.) - teologie osvobození n. f.

théologie sacramentaire $\mathrm{n}$. f. (th.) - sakramentologie $\mathrm{n}$. $\mathrm{f}$.

tiers ordre n. m. (pr.) - třetí řád n. m.

tonsure $\mathrm{n}$. f. (lit.) - tonzura $\mathrm{n}$. $\mathrm{f}$.

tourier, ère n. m., n. f. (pr.) - X

urbi et orbi loc. adv. (mag.) - urbi et orbi loc. adv.

vertus cardinales $\mathrm{n}$. f. pl. (th. mor.) - kardinální ctnosti n. pl. f.

vicaire épiscopal n. m. (CIC) - biskupský vikář n. m.

vicaire forain n. m. (CIC) - okrskový vikář n. m., děkan n. m. (epsé)

vicaire général n. m. (CIC) - generální vikář n. m.

vicaire paroissial $\mathrm{n}$. $\mathrm{m}$. (CIC) - farní vikář n. m.

vicariat apostolique n. m. (CIC) - apoštolský vikariát n. m.

\section{Conclusion}

Nous appelons un terme propre à un domaine de spécialité (dans ce cas au domaine de la religion catholique) le terme "endémique ». En accord avec la conception du souscode, ce terme appartient au système des termes du domaine concerné. Ni son signifiant ni son signifié ne sont propres à d'autres domaines de spécialité. En conséquence, ce sont uniquement les termes " endémiques » à un domaine de spécialité qui désignent exactement un concept même hors du contexte. Néanmoins la synonymie et la polysémie peuvent apparaître au sein des termes « endémiques ». Un terme " endémique » au catholicisme désigne un concept propre à la religion catholique. Il ne doit pas être employé exclusivement dans les textes du domaine concerné.

Grâce aux dictionnaires, aux textes de spécialité et au corpus parallèle informatisé InterCorp nous avons répertorié une liste de 131 unités terminologiques françaises classifiées comme « endémiques » au catholicisme.

Nous avons constaté que tous les équivalents de ces termes ne sont pas de même « endémiques » en tchèque. Dans ce cas-là, les équivalents non «endémiques » ne vont pas désigner un concept de manière univoque sans l'aide du contexte. C'est de même un attribut apte à spécifier un tel équivalent qui va éliminer le risque de l'ambiguïté.

\footnotetext{
Abréviations

CEC - Catéchisme de l'Église catholique

CIC - Code de droit canonique

epsé - terme « endémique » au catholicisme par un de ses signifiés

KKC - Katechismus katolické církve

lit. - liturgie

mag. - magistère de l'Église, papauté

ne - terme « non endémique » au catholicisme

pr. - pratique pastoral, domaine des religieux

th. - théologie
} 


\section{Bibliographie}

ANZENBACHER, Arno (2004), Křestáanská sociální etika: úvod a principy, Brno : Centrum pro studium demokracie a kultury.

BERGER, Rupert (2008), Liturgický slowník, Praha : Vyšehrad.

Catéchisme de l'Église catholique (1998), Paris: Centurion/Cerf/Fleurus-Mame/ Libraire Editrice Vaticane.

CABRÉ, Maria Teresa (1998), Terminologie: théorie, méthode et applications. Paris: Armand Colin.

Concil Vatican II (1965), Gaudium et Spes. Constitution pastorale sur l'Église dans le monde de ce temps, version éléctronique, http://www.vatican.va/archive/hist_ councils/ii_vatican_council/documents/vat-ii_cons_19651207_gaudium-etspes_fr.html, en ligne le 29 mai 2013.

Dictionnaire Hachette langue française (2001), Paris : Hachette.

Dubuc, Robert (2002), Manuel pratique de la terminologie, Brossard : Linguitech.

Feuillet, Michel (2010, $3^{\mathrm{e}}$ éd., $2^{\mathrm{e}}$ tirage), Vocabulaire du christianisme, Paris : Presses Universitaires de France.

FiLIPI, Pavel (1998), Křstáanstvo. Historie, statistika, charakteristika křestanských církví, Brno : Centrum pro studium demokracie a kultury.

FiLIPI, Pavel et al. (2008), Malá encyklopedie evangelických církví, Praha : Libri.

FisCHER-WollPERT, Rudolf (1995), Malý teologický slovník: přehled papežů, Praha : Zvon.

Franzen, August (1995), Malé církevní dějiny, Praha : Zvon.

Grand Robert de la langue française (2005), version électronique, [CD-ROM], Le CD -ROM du Grand Robert, version 2.0, Paris : Le Robert / SEJER.

GuERARD, Françoise, éd. (1989), Le dictionnaire de notre temps 1990, Paris : Hachette.

HavráneK, Bohuslav, éd. (1989), Slovník spisovného jazyka českého. 1 - 8. Version électronique, http:/ / ssjc.ujc.cas/cz, en ligne le 29 mai 2013.

InterCorp (2013, avril 8, version 6), http:/ / www.korpus.cz/intercorp, en ligne le 29 mai 2013.

Katechismus katolické církve (2001), Karmelitánské nakladatelství, Kostelní vydří. (KKC).

Kodex kanonického práva : úřední zněni textu a překlad do češtiny : latinsko - české vydání s věcným rejstřikem (1994), Praha: Zvon.

Kraus, Jiří et al. (2005), Nový akademický slovník cizích slov, Praha : Academia.

Le Tourneau, Dominique (2002,3éd.), Ledroit canonique, Paris :Presses universitaires de France.

Le Tourneau, Dominique (2005), Les mots du christianisme. Catholicisme, orthodoxie, protestantisme, Paris : Fayard.

Lexique orthodoxe (2004, avril4), versionélectronique, http:/ / www.pagesorthodoxes. net/ressources/lexique.htm, en ligne le 5 novembre 2012.

MACHOVÁ, Svatava (1995), « Terminografie », in : ČERMÁK, František - BlatNÁ, Edita, éds. : Manuál lexikografie, Jinočany : Nakladatelství a vydavatelství H\&H, 137-157.

Raner, Karl - Vorgrimler, Herbert (1970), Petit dictionnaire de théologie catholique, Paris : Éditions du Seuil.

RoELCKE, Thorsten (1999), Fachsprachen, Berlin : Erich Schmidt. 
ŠAbršula, Jan - SvobodovÁ, Jitka - KRejzovÁ, Anna (1983), Základy jazykovědy pro romanisty, Praha : Univerzita Karlova.

ŠABRŠULA, Jan (1985), Substitution, représentation, diaphore, Praha : Univerzita Karlova.

ŠIMANDL, Josef et al. (2004), Jak zacházet s náboženskými výrazy: pravopis, výslovnost, tvary, význam, Praha: Academia.

Wikipédia l'encyclopédie libre. Anglicanisme (2013, mai 24), http:/ / fr.wikipedia.org/ wiki/Anglicanisme, en ligne le 29 mai 2013.

Pavlína Žídková

Katedra romanistiky

Filozofická fakulta

Ostravská univerzita v Ostravě

Reální 5

70103 Ostrava

République tchèque

pavlina.zidkova@osu.cz 